\title{
À la recherche de la mémoire des Femmes dans la Guerre
}

\section{Notes méthodologiques}

Looking for the memory of Women in war: methodological notes

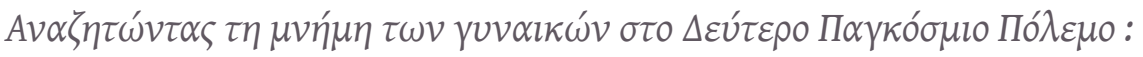

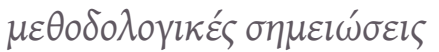

\section{Maria Thanopoulou}

\section{(2) OpenEdition}

Journals

Édition électronique

URL : https://journals.openedition.org/ceb/3951

DOI : 10.4000/ceb.3951

ISSN : 2261-4184

Éditeur

INALCO

Édition imprimée

Pagination : 221-228

ISBN : 978-2-85837-205-4

ISSN : 0290-7402

\section{Référence électronique}

Maria Thanopoulou, « À la recherche de la mémoire des Femmes dans la Guerre », Cahiers balkaniques [En ligne], 41 | 2013, mis en ligne le 16 mai 2013, consulté le 06 juillet 2021. URL : http:// journals.openedition.org/ceb/3951 ; DOI : https://doi.org/10.4000/ceb.3951

Ce document a été généré automatiquement le 6 juillet 2021.

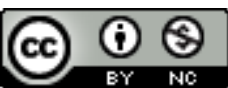

Cahiers balkaniques est mis à disposition selon les termes de la Licence Creative Commons Attribution - Pas d'Utilisation Commerciale 4.0 International. 


\title{
À la recherche de la mémoire des Femmes dans la Guerre
}

\author{
Notes méthodologiques \\ Looking for the memory of Women in war: methodological notes

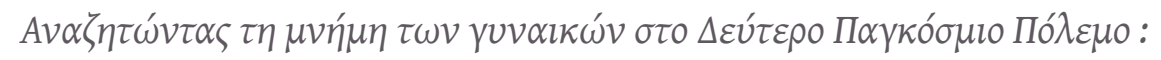

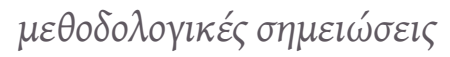

Maria Thanopoulou

1 Aborder la mémoire des femmes sous la Seconde Guerre mondiale est en fait tenter de déplacer l'axe de l'intérêt porté sur l'histoire du Front et des batailles à une histoire de l'arrière, d'une histoire d'hommes-soldats à une histoire de femmes assurant la continuité de la vie quotidienne, en même temps que subissant les conséquences de la guerre $^{1}$. Un champ de recherche vaste et étendu s'ouvre devant nous, un champ qui doit, à chaque fois, être délimité par rapport à l'objectif de la recherche. Plusieurs questions d'ordre méthodologique se posent, questions qui, dans un cadre d'enquête précis, pourraient servir à délimiter le champ de recherche. En partant du cas de la Grèce, pays qui dispose d'une longue et douloureuse histoire d'épreuves pendant la Seconde Guerre mondiale, nous essayons de démontrer combien il est complexe de planifier une recherche visant à l'étude de la mémoire des femmes. En fait, nous mettons l'accent sur la recherche de cette la mémoire orale, mémoire qui se transmet surtout de bouche à oreille et qui est rarement transcrite au sein d'une enquête ou invoquée lors de l'écriture d'un témoignage. Nous ne traitons pas de la recherche sur la mémoire des femmes incluse dans les témoignages écrits des années $40^{2}$, autre forme de survivance et d'alimentation de la mémoire sur la guerre, car elle est issue d'un processus de transformation de la mémoire orale en mémoire écrite et aboutit à une mémoire figée et objectivée. D'ailleurs les femmes témoignent moins à ce sujet que les hommes, soit parce qu'elles se sentent moins légitimes pour écrire sur des affaires masculines ${ }^{3}$ - comme la guerre -, soit parce qu'elles possèdent moins l'aptitude de déposer un témoignage écrit de leur mémoire sur le vécu de la guerre ${ }^{4}$ et qu'elles sont surtout porteuses de la tradition orale ${ }^{5}$. La focalisation sur la mémoire orale des femmes relève d'un souci de suggérer certains points importants quant à la recherche 
de la mémoire orale en général, à un moment où les jeunes chercheurs, charmés par les outils de la recherche qualitative et les perspectives qui s'ouvrent à travers l'enquête orale, tendent parfois à utiliser ces outils sans se rendre compte du processus de stimulation de la mémoire que leurs choix méthodologiques déclenchent. Le fil de notre réflexion se déploie autour de trois questions interdépendantes qui nous paraissent importantes quant au déroulement d'une enquête orale.

\section{Quel objectif sous-tend cette recherche de la mémoire orale des femmes pendant la guerre et régit le fil conducteur de l'enquête orale?}

2 Il se peut que l'objectif de la recherche soit rattaché à celui d'une enquête d'histoire orale qui vise à découvrir et à créer de nouvelles sources historiques. Dans ce cas, il s'agirait d'une recherche qui viserait à utiliser la mémoire des femmes pendant la guerre comme outil ou source permettant au chercheur d'accéder à un passé éloigné dont survivent encore quelques témoins. Dans le cadre d'une telle enquête, enquête relevant de l'histoire orale, ce qui serait important, serait la recherche de la mémoire de femmes qui pourraient être considérées comme témoins privilégiés par rapport à l'objet d'étude, ce qui, à son tour, renverrait à un réseau de témoins ou bien à des réseaux sociaux ayant accès à des témoins appropriés. Dans le cas d'une enquête d'histoire orale, le fil conducteur de la démarche du chercheur est déployé avec le souci de la sauvegarde du passé, de la fiabilité des témoins et de la validité des témoignages oraux obtenus. Ce qui est en cause, c'est la relation entre histoire et mémoire, relation qui est sous-jacente tout au long du processus de la collecte des témoignages oraux, ainsi que pendant leur analyse. Ce qui compte est le passé de la guerre et sa restitution plus ou moins fidèle et fiable.

3 Il se peut également que l'objectif de la recherche de la mémoire orale des femmes dans la guerre soit celui d'une enquête sociologique sur la mémoire qui porterait sur le processus social de reconstruction du passé de la guerre à partir du présent, et sur les usages sociaux de cette reconstruction. Dans le cadre d'une telle enquête, la mémoire orale des femmes pourrait être l'objet même de la recherche en tant que fragment de la mémoire collective structurée ${ }^{6}$ et différenciée selon le sexe des membres de la collectivité étudiée. Ce qui serait important dans le cas d'une enquête sociologique sur la mémoire des femmes serait alors la recherche sur les réseaux sociaux auxquels les femmes interviewées appartiennent, réseaux contribuant à la remémoration du passé de la guerre, et à sa survivance dans le présent, à travers le travail de stimulation et d'alimentation de la mémoire de la guerre qu'ils effectuent. Dans le cas d'une enquête sociologique sur la mémoire des femmes sous la guerre, le fil conducteur de l'enquête orale est déployé avec le souci de dépister les cadres sociaux de la mémoire (temps, espace, collectivités, rites, etc.), cadres rendant le rappel possible ${ }^{7}$, ou de dépister les enjeux sociaux actuels se rattachant à la remémoration du passé de la guerre. Ce qui est en cause est la relation entre la société et la mémoire de la guerre, le rapport de la société à son passé établi à travers les divers mécanismes sociaux de fabrication et de conservation de la mémoire, l'usage même que la société fait du passé de la guerre. Ce qui compte n'est plus le passé, c'est le présent comme point de départ de toute reconstruction de la mémoire orale à l'aide des données empruntées au présent ${ }^{8}$, mais 
aussi comme temps crucial au sein duquel survit le passé sous forme de mémoire. Ce qui compte est la survivance du passé de la guerre dans le présent.

4 À travers l'esquisse de deux types d'enquête orale différenciés par leur objectif, nous visons à signaler que la question cruciale qui se pose dès le début de la recherche est la suivante : Qui s'intéresse à la mémoire des femmes sous la guerre un demi-siècle plus tard et pourquoi ? C'est une question dont la réponse renvoie à l'identité même du chercheur, ainsi qu'à ses motifs. ${ }^{9}$

\section{Qu'est-ce que la « mémoire des femmes », qui la porte?}

Lorsque nous nous engageons dans la recherche de la mémoire orale des Femmes pendant la guerre, il va de soi que nous devons nous adresser aux femmes mêmes afin de dépister le côté féminin - non connu ou caché - de cette expérience collective. Un demi-siècle après la guerre, pouvons-nous interviewer des survivantes qui soient à même - du point de vue de leur santé mentale ${ }^{10}$ - de se rappeler la guerre ? Et si elles ne sont plus en vie, devons-nous nous adresser à leurs filles - la génération d'aprèsguerre - qui sont éventuellement porteuses d'une mémoire féminine qui leur a été oralement transmise par leurs mères ${ }^{11}$ ? Et si nous recourons à la mémoire transmise de la mère aux enfants, pourquoi les fils ne seraient-ils aussi porteurs des fragments de la mémoire de leur mère sur l'expérience de la guerre? La mémoire évoquée dans un témoignage oral est issue du vécu personnel ou provient d'une transmission de bouche à oreille, elle comporte des fragments de mémoires collectives rattachées à la vie et à l'expérience des collectivités auxquelles appartiennent des narrateurs ayant transmis leurs souvenirs.

Puis, il y aurait une mémoire de la guerre par couche sociale, ce qui implique que nous devrions nous adresser à des femmes appartenant à diverses couches sociales, non seulement à l'époque de la guerre, mais aussi dans le présent, temps de l'enquête ; il se peut en effet qu'une mobilité sociale ascendante ou descendante ait changé le statut social de la femme interviewée, ce qui aurait une incidence directe au processus même du rappel, ainsi qu'au contenu des souvenirs évoqués.

7 Engagés dans la recherche de la mémoire des femmes, devons-nous nous adresser uniquement aux femmes ou pouvons-nous nous adresser aussi aux hommes? Visonsnous une complémentarité, c'est à dire contre-croiser la mémoire des femmes avec celle des hommes, ou nous cherchons-nous à comprendre comment la mémoire des femmes a pu être exclue ou marginalisée au sein des sociétés d'avant-guerre, sociétés à dominance masculine? Ou notre attention porte-t-elle sur ce qui se passe actuellement quant à la survivance de la mémoire des femmes dans un univers mémoriel probablement dominé par les hommes?

8 Lorsque nous nous engageons dans un processus de recherche de la mémoire des femmes, une deuxième question cruciale se pose : sur quels critères seront reconnus et choisis les porteurs de la mémoire des femmes sous la guerre, porteurs-candidats à l'interview au sein d'une enquête orale? La détermination de ces critères conduira par la suite l'orientation de l'enquête orale, ainsi que les attentes du chercheur quant au déploiement de la mémoire des interviewés. 


\section{Quel matériel mnémonique à la suite d'une enquête orale?}

9 La mémoire orale recueillie lors de l'interview est une mémoire adressée au chercheur, donc à un récepteur concret qui a stimulé la mémoire individuelle du narrateur par des questions dictées par l'objet de sa recherche. Il s'agit d'une mémoire transcrite à un moment donné, dans des conditions données (conjonctures sociales et politiques, moment spécifique de la trajectoire de l'interviewé et du chercheur). Il y a un présent, un point de départ temporel qui régit et influence la sélection des souvenirs évoqués. C'est pourquoi ce que nous obtenons comme matériel mnémonique porte les traces de la situation de l'interview et de l'interaction effectuée entre le chercheur et le narrateur. Ceci implique que ce que nous obtenons ne représente pas nécessairement la totalité des souvenirs que l'interviewé possède dans sa mémoire individuelle au sujet de vécu collectif de la guerre, mais seulement une partie de celui-ci, celle évoquée lors du rappel provoqué par les questions du chercheur. Le reste de ce passé (mémoire défendue, traumatique, etc.) peut être livré au silence ou à l'oubli. D'autre part, il faudrait signaler que ce que nous obtenons comme matériel mnémonique n'est pas forcément le même quant au contenu et à la forme, que la mémoire orale de la guerre qui circule parmi les membres des réseaux sociaux auxquels l'interviewé participe. $\mathrm{Au}$ sein de ces réseaux (réseaux d'anciens camarades, d'amis, etc.) la mémoire individuelle s'imbrique dans les mémoires orales des autres membres qui la stimulent et l'alimentent. Il se peut que cette mémoire circulant entre les membres du réseau soit une mémoire stéréotypée, centrée autour des actes héroïques, des vécus de martyre, des incidents illustrant les côtés dramatiques de l'expérience collective de la guerre, une mémoire-preuve combattant la falsification de la vérité ou l'oubli. Il y aurait alors à faire toute une autre étude sur la mémoire des femmes dans la guerre, si nous visions à considérer cette mémoire par rapport aux processus de fabrication, de conservation et d'alimentation de la mémoire collective d'une collectivité ou d'une communauté ${ }^{12}$.

Lors de la recherche de la mémoire des femmes dans la guerre, il ne faudrait pas oublier que ce que nous obtenons n'est pas une description du passé même; c'est le produit ou la composante des reconstructions antérieures effectuées tout au long du temps à partir de données empruntées à diverses époques. Ce que nous obtenons est une image du passé altérée ${ }^{13}$. De même, il ne faudrait pas oublier le travail de sélection constamment entrepris par la mémoire individuelle, qui fait que la mémoire obtenue lors de l'interview porte également les traces de ce travail de sélection, ce qui fait que le rappel de certains souvenirs implique en même temps le passage sous silence ou l'oubli de certains autres ${ }^{14}$.

11 Lorsque nous sommes à la recherche de la mémoire des femmes dans la guerre, une autre question cruciale se pose : comment délimiter le champ de la recherche de sorte à pouvoir fixer bien clairement les limites mêmes de la démarche entreprise ? En fait, en mettant l'accent sur la recherche du passé même, ou sur la survivance de ce passé dans le présent, nous parvenons à un choix décisif quant à la place que la mémoire tiendra au sein de cette démarche, choix qui régit par la suite la façon dont nous abordons le matériel obtenu lors de l'enquête orale. 


\section{Remarques finales}

12 Ces quelques notes méthodologiques visent à démontrer que la recherche de la mémoire orale des femmes dans la guerre est un processus de recherche dynamique qui se développe en fonction de la perspective du chercheur et implique des choix constants qui doivent être justifiés par rapport à l'objectif de la recherche. C'est pourquoi il n'est pas possible d'étudier le contenu de la mémoire orale des femmes dans la guerre, sans prendre en considération les conditions sociales, spatiales et temporelles qui ont éventuellement contribué à une marginalisation de cette mémoire pendant la période d'après-guerre. De même il n'est pas possible d'étudier cette mémoire dans le présent sans prendre en compte les conditions sociales, spatiales et temporelles qui favorisent actuellement l'émergence de cette mémoire dans le présent et plus particulièrement le déroulement des enquêtes orales sur la mémoire des femmes sous la guerre.

\section{BIBLIOGRAPHIE}

Augé, Marc, (1998), Les formes de l'oubli, Paris : Manuels Payot.

Bertaux-Wiame, Isabelle, (1985), « Mémoire et récits de vie », Pénélope, Mémoire de femmes, 12, p. 47-54.

Burgchardt, Natasha, (1993), “Transgenerational transmission in the families of Holocaust survivors in England", in Daniel Bertaux and Paul Thompson (eds), Between Generations. Family models, myths and memories, Oxford: Oxford University Press, pp. 121-138.

Derouesné Christian et Poitier Jean, (1985), « La mémoire malade du cerveau », Pénélope, Mémoire de femmes, 12, p. 19-23.

Devreux, Anne-Marie, (1985), « La mémoire n'a pas de sexe », Pénélope, Mémoire de femmes, 12, p. 55-68.

Halbwachs, Maurice, (1967), La mémoire collective, $2^{\mathrm{e}}$ éd., Paris : PUF, p. 57-58.

Halbwachs, Maurice, (1975), Les cadres sociaux de la mémoire, Paris : Mouton, p. 146-168.

Papathanassiou, Joanna, (1996), « Vécu, Histoire et Politique. Le fondement du témoignage

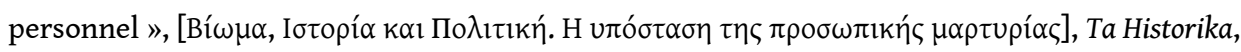
24-25, p. 253-256.

Papathanassiou, Joanna, (2007), « Usage et abus des sources ou l'Histoire en tant qu'impression »,

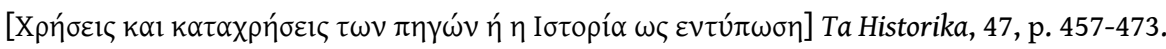

Papathanassiou, Joanna, (2005), « Mémoires d'une décennie. Mémoires de la Gauche. L'itinéraire

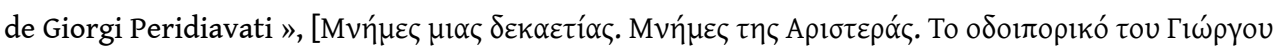

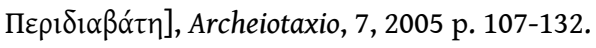

Papathanassiou, Joanna, (2007), « Usage et abus des sources ou l'Histoire en tant qu'impression »,

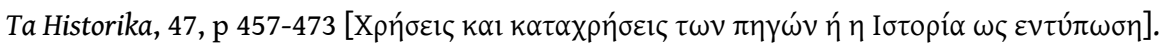


Pollak, Michel, (1985), « Encadrement et silence : Le travail de la mémoire », Pénélope, Mémoire de femmes, 12, 1985, p. 35-39.

Rioux, Jean-Pierre, (1985), « À la recherche de la mémoire », Pénélope, Mémoire de femmes, 12, p. 153-162.

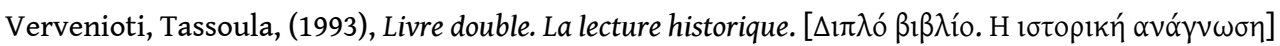
Athènes : Vivliorama.

Yannissopoulou, Maria, Thanopoulou, Marie, Leontsini, Mary, (2006), « Du témoignage écrit à la

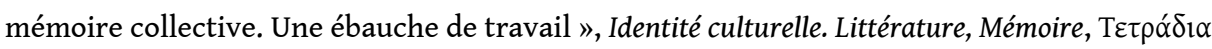
$\varepsilon \rho \gamma \alpha \sigma i ́ \alpha \varsigma, 30$, Athènes : Institut de Recherche Néohelléniques, Fondation Nationale de la Recherche scientifique, p. 185-193.

\section{NOTES}

1. Ce texte constitue une nouvelle version plus élaborée de la communication présentée lors du Colloque international «Femmes des Balkans sous l'Occupation » organisé par l'INALCO le 9 et 10 décembre 2011.

2. Joanna Papathanassiou, «Vécu, Histoire et Politique. Le fondement du témoignage

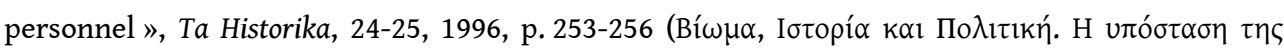

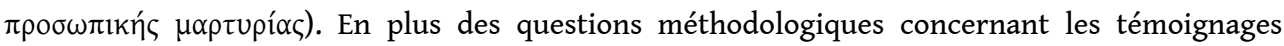
écrits sont traités dans l'article du même auteur, voir Joanna Papathanassiou « Usage et abus des

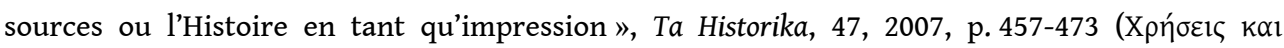

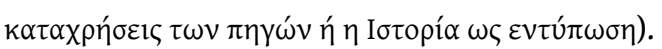

3. Joanna Papathanassiou, «Mémoires d'une décennie. Mémoires de la Gauche. L'itinéraire de

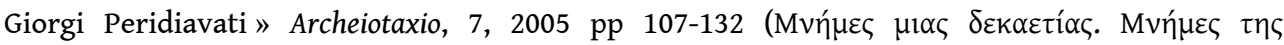

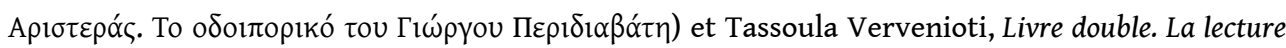

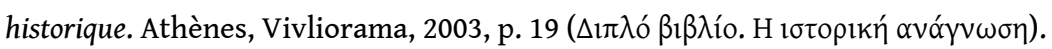

4. Maria Yannissopoulou, Marie Thanopoulou, Mary Leontsini, «Du témoignage écrit à la

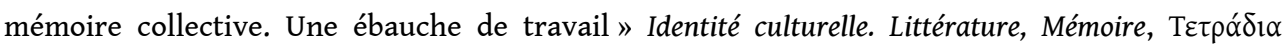

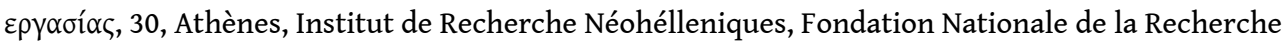
scientifique, 2006, p. 185-193.

5. Anne-Marie Devreux, «La mémoire n’a pas de sexe », Pénélope, Mémoire de femmes, 12, 1985, p. 55-68.

6. Maurice Halbwachs, La mémoire collective, $2^{\mathrm{e}}$ éd., Paris, PUF, 1967, p. 57-58.

7. Maurice Halbwachs, Les cadres sociaux de la mémoire, Paris, Mouton, 1975, p. 146-168.

8. Maurice Halbwachs, La mémoire collective, op.cit., p. 57-58.

9. Jean-Pierre Rioux, « À la recherche de la mémoire », Pénélope, op.cit., p. 153-162.

10. C. Derouesné et J.Poitier, « La mémoire malade du cerveau », Pénélope, op.cit., p. 19-23.

11. Natasha Burgchardt, «Transgenerational transmission in the families of Holocaust survivors in England », Daniel Bertaux and Paul Thompson (eds), Between Generations. Family models, myths and memories, Oxford, Oxford University Press, 1993, p. 121-138.

12. Michael Pollak, « Encadrement et silence : Le travail de la mémoire », Pénélope, op.cit., p. 35-39.

13. Maurice Halbwachs, La mémoire collective, op.cit., p.57-59 et Isabelle Bertaux-Wiame, "Mémoire et récits de vie ", Pénélope, op.cit., p. 47-54.

14. Marc Augé, Les formes de l'oubli, Paris, Manuels Payot, 1998. 


\section{RÉSUMÉS}

La recherche sur la mémoire des femmes dans la Seconde Guerre mondiale offre sur un champ riche et étendu qui doit être délimité. Avec, comme point de départ de notre réflexion, le cas de la Grèce, en mettant l'accent sur la recherche de la mémoire orale, nous abordons quelques questions méthodologiques qui pourraient servir à délimiter ce champ. Quel objectif sous-tend cette recherche et régit le fil conducteur de l'enquête orale ? Qu'est la "mémoire des femmes ", quelles femmes en sont porteuses? Qui interroger et comment? Quel matériel mnémonique obtenons-nous à la suite d'une enquête orale ? Par ce type de questions, nous visons à montrer que la recherche sur la mémoire des femmes dans la guerre est un processus dynamique dont les traces sont vivement imprégnées du matériel recueilli au cours de l'enquête. Ainsi est-il impossible d'étudier le contenu de cette mémoire sans prendre en considération les conditions sociales, spatiales et temporelles qui en ont favorisé l'émergence et le recueil et ont influencé sa forme.

Research on memory of Women in WWII offers a rich and extensive field that needs to be delimited. With Greece, as starting point of our reflection, and focusing on oral memory, we try to deal with some methodological questions which could help us to delimit this research field. What is the purpose of this research, the leading thread of this oral investigation? What is "memory of Women"? Who is carrying it? Whom should we ask and how? What mnemonic equipment have we collected in the end of one oral investigation? With such questions, we aim at showing that research about the memory of women in War is a dynamic process whose traces are soaked with the results of the oral inquiry. Thus, it is impossible to study the results of such inquiry without considering the social, chronological and spatial conditions that have promoted their birth and collection and influenced their form.

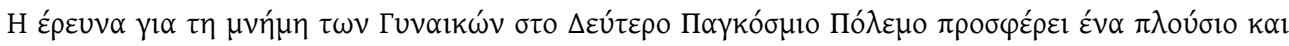

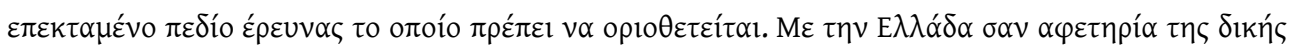

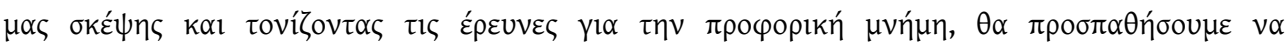

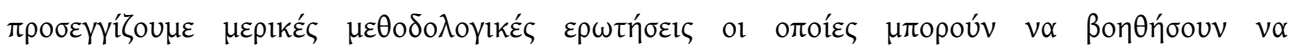

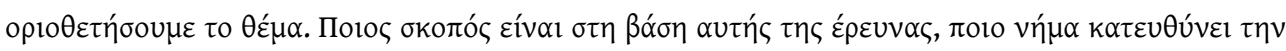

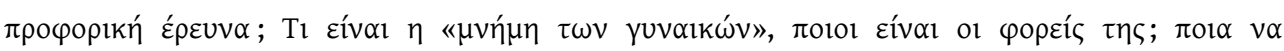

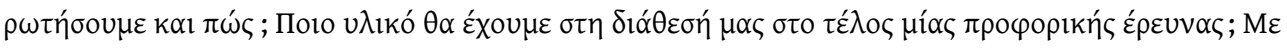

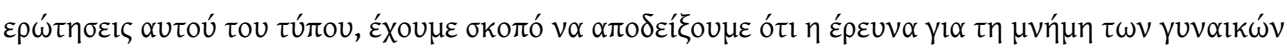

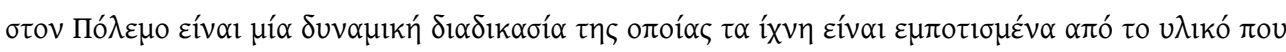

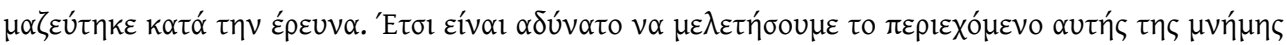

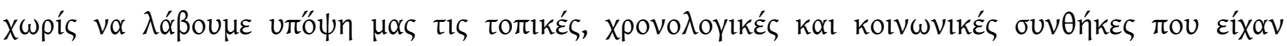

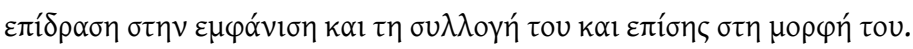


INDEX

Index géographique : Grèce

motsclesmk МЕМОРИЈА ЖЕНИ, ОРАЛЕН МЕМОРИЈА, ОРАЛЕН ИСТРАЖУВАҢЕ, МЕТОДОЛОГИЈА, СЕЌАВАњЕТО НА ВТОРАТА СВЕТСКА ВОЈНА, ИСТОРИЈА, МЕТОДОЛОГИЈА НА ИСТОРИЈАТА

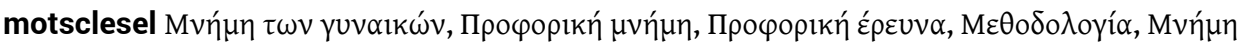

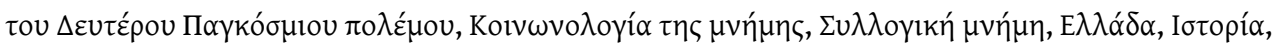

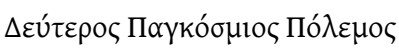

motsclestr Bellek kadın, Oral bellek, Sözlü Araştırma, Metodoloji, İkinci Dünya Savaşı anısına, Tarih, Tarihin metodolojisi

Mots-clés : mémoire des femmes, mémoire orale, enquête orale, méthodologie, mémoire collective

Keywords : Collective memory, Methodology, Women memory, Oral memory, Greece, Oral research, History

Thèmes : Histoire, Historiographie

Index chronologique : guerre mondiale (1939-1945) 